\title{
Excitation of low-frequency dust density waves in flowing complex plasmas ${ }^{\circ}$
}

Cite as: Phys. Plasmas 26, 053702 (2019); https://doi.org/10.1063/1.5097128

Submitted: 22 March 2019 . Accepted: 25 April 2019 . Published Online: 14 May 2019

V. V. Yaroshenko, S. A. Khrapak (D), M. Y. Pustylnik, H. M. Thomas (D), S. Jaiswal, A. M. Lipaev, A. D. Usachev, O. F. Petrov, and V. E. Fortov

\section{COLLECTIONS}

EP

This paper was selected as an Editor's Pick
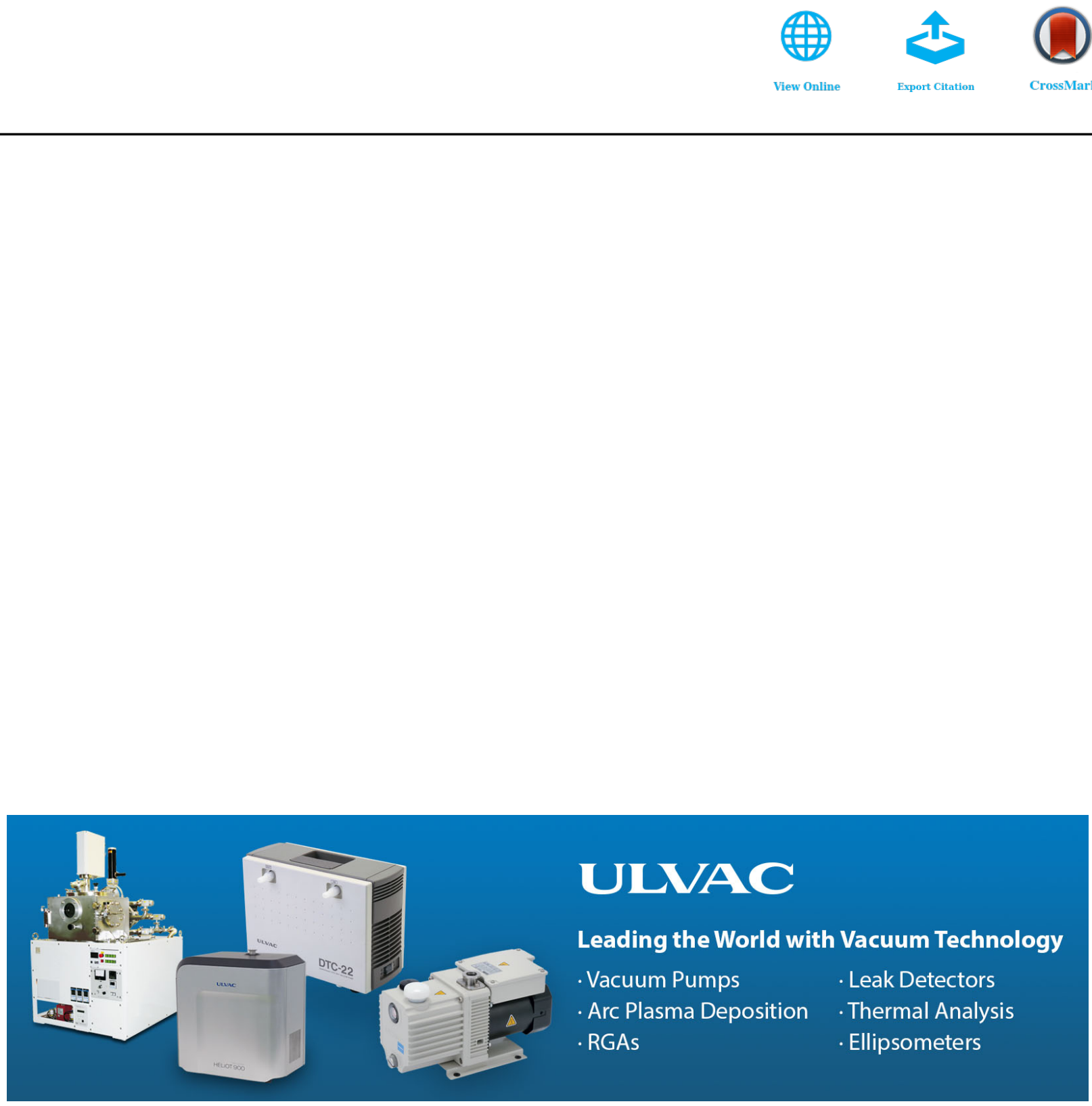


\title{
Excitation of low-frequency dust density waves in flowing complex plasmas (ip
}

\author{
Cite as: Phys. Plasmas 26, 053702 (2019); doi: 10.1063/1.5097128 \\ Submitted: 22 March 2019 - Accepted: 25 April 2019 . \\ Published Online: 14 May 2019
}

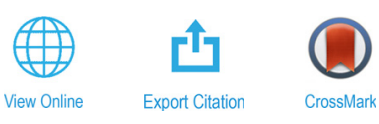

V. V. Yaroshenko, ${ }^{1, \text { a) }}$ S. A. Khrapak, ${ }^{1}$ (D M. Y. Pustylnik, ${ }^{1}$ H. M. Thomas, ${ }^{1}$ iD S. Jaiswal, ${ }^{2}$ A. M. Lipaev, ${ }^{3}$ A. D. Usachev, ${ }^{3}$ O. F. Petrov, ${ }^{3}$ and V. E. Fortov ${ }^{3}$

\author{
AFFILIATIONS \\ 'Institut für Materialphysik im Weltraum, Deutsches Zentrum für Luft- und Raumfahrt (DLR), 82234 Wessling, Germany \\ ${ }^{2}$ Physics Department, Auburn University, Auburn, Alabama 36849, USA \\ ${ }^{3}$ Joint Institute for High Temperatures, Russian Academy of Science, Izhorskaya st. 13 Bd.2, Moscow 125412, Russia \\ a)Electronic mail: Victoriya.Yaroshenko@dlr.de
}

\begin{abstract}
We study low frequency dust density waves excited in the microparticle streams of the Plasmakristall-4 chamber under microgravity conditions on board the International Space Station. Dispersion relations and instability conditions are analyzed for the dust density modes propagating along the axial electric field and in the reversed direction, revealing some important implications for the dust diagnostics. The results clearly point out to the Havnes parameter as a key factor for the excitation of the dust density modes.
\end{abstract}

Published under license by AIP Publishing. https://doi.org/10.1063/1.5097128

\section{INTRODUCTION}

Dust density wave observations play a key role in dusty (complex) plasma research. The waves are usually spontaneously excited over a wide range of the plasma/discharge conditions (see the review and references therein ${ }^{1}$ ). Applications include wave phenomena in processing plasmas, laboratory complex plasmas, and astrophysical phenomena. ${ }^{1-3}$ Some of the dust density modes resemble the dustacoustic wave $(\mathrm{DAW})^{4}$ but were explained through the recognition of the role of ion drift effects. ${ }^{5,6}$ A common feature of the experimental studies of the dust density modes is the presence of weak electric fields on the order of a few volts per centimeter. It is usually assumed that the electric fields produce the ion drift that provides the energy for the wave excitation. Many aspects of ion-dust streaming instabilities under various laboratory conditions have been discussed for several decades but most studies have not considered the dust particle equilibrium drifts. $^{7-12}$ The Plasmakristall-4 (PK-4) facility on board the International Space Station (ISS) allows for the direct electric field manipulation of the dust dynamics. In particular, the first direct observations of the dust modes propagating in the particle streams at the discharge polarity reversal ${ }^{13}$ look very promising in furthering our knowledge about collective processes in dusty (complex) plasmas.

In this paper, we report on the theoretical interpretation of such dust-density waves observed in the PK-4 chamber at alternative discharge polarity in the presence of neutral gas flow. It is remarkable that perturbations at negative and positive polarities propagate in the same direction and exhibit almost acousticlike dispersion in the longwavelength limit. At the same time, the wave modes revealed different phase velocities. Our study deals with the modification of the collective modes due to the equilibrium dust drifts in the external electric fields, which is typically irrelevant for other wave experiments (in many studies, the dust particles are stationary due to the balance of various forces, like the electrostatic and gravitational, ion and neutral drag force, etc.). Our aim is, therefore, to give an explanation for the onset of the self-excited drift waves in terms of a linear stability analysis for the PK- 4 conditions and to discuss the implications for the dust diagnostics.

\section{EXPERIMENTAL OBSERVATIONS}

The experiment was performed in the flight model of the Plasmakristall-4 facility (see Refs. 13 and 14 for more details about the original device) under microgravity conditions on board the ISS. The dc discharge was operated in neon gas at a gas pressure of $40 \mathrm{~Pa}$. In the experiment, the valve of the flow controller was closed, but a small gas leak of about $0.1 \mathrm{sccm}$ was observed. The injected monodisperse melamine formaldehyde microparticles of radius $a \approx 1.7 \mu \mathrm{m}$ were first trapped in an inductively coupled plasma $(0.4 \mathrm{~W}$ power produced by a movable rf coil) and then released to drift for some seconds in a dc discharge with a constant negative current $\left(j_{d c}=-0.5 \mathrm{~mA}\right)$. After a few seconds, the discharge polarity was reversed $\left(j_{d c}=+0.5 \mathrm{~mA}\right)$. In both cases, the self-excited low frequency $(\$ 10 \mathrm{~Hz})$ modes were observed. ${ }^{13}$ 
The dust dynamics was visualized by illuminating the particles with a green laser light and imaging with two digital video cameras with a total field of view of $44 \times 7 \mathrm{~mm}^{2}$ and the resolution of about $14 \mu \mathrm{m} /$ pix. The frame rate of recording was $70 \mathrm{fps}$. In Fig. 1, we show the spatiotemporal pattern of the scattered light intensity $J(x, t)$ [supposed to be proportional to the local microparticle number density $\left.n_{d}(x, t)\right]$. The latter has been obtained by combining images from two cameras and averaging the intensities over 7 pixels in a narrow rectangular region across the wave crests as indicated in Fig. 1(a). The wave pattern reveals the inhomogeneous microparticle drift velocity (that drops from a head to tail of the dust cloud) and bifurcations after the polarity reversal. ${ }^{13}$ Therefore, our further analysis is restricted to the regions in the cloud tails that are closer to the free-dust discharge and do not reveal bifurcations [see marked rectangles in Fig. 1(b)]. We have calculated the fast Fourier transform using the pixel intensity of raw experimental images of waves $J(x, t)$ in the space and time domain indicated in Fig. 1(b). The resulting spectra of fluctuations $I^{2}(k, \omega)$ represent the distribution of wave energy in the wave number-wave frequency plane are shown in Figs. 2(a) and 2(b), for the negative/ positive polarity, respectively. By inspection of the spectra $I^{2}(k, \omega)$, we can clearly identify the frequencies and wave numbers for the most unstable mode appearing at $k \leqq 40 \mathrm{~cm}^{-1}$ [shown in Figs. 2(c) and 2(d)], which will be later associated with theoretical dispersion relations and discussed below. Although the fundamental mode is clearly visible at the negative and the positive polarities, several harmonics generated by the nonlinear effects are also seen in Figs. 2(a) and 2(b). The enhanced fluctuations of the wave energy at large wave numbers $\left(k>40 \mathrm{~cm}^{-1}\right)$ seem to be mainly due to the generation of such high harmonics.

The observed properties of the fundamental wave modes [Figs. 2(c) and 2(d)] can be summarized as follows: (i) The modes are effectively excited in the range of wave numbers $15 \mathrm{~cm}^{-1} \lesssim k$ $\lesssim 35 \mathrm{~cm}^{-1}$, i.e., at long-wavelength range $k \Delta \sim k \lambda_{D i}<1$ (here $\Delta$ is the average interparticle distance and the ion Debye length $\lambda_{D i} \sim 10^{-2} \mathrm{~cm}$ ); (ii) small wave number cutoffs at $k_{1} \sim 1-5 \mathrm{~cm}^{-1}$ are seen at the both dependencies; (iii) maximum of the wave energy spectrum $I^{2}(k, \omega)$ identifies the most unstable wave numbers $k_{\max } \sim 20 \mathrm{~cm}^{-1}$ in both cases; (iv) although both of the wave modes remain close to the acoustic type, different discharge polarities manifest slightly different dispersion properties. For negative polarity, the propagation direction is opposite to the electric field, and the perturbations show generally higher frequencies and larger phase velocities than the mode aligned with the electric field in the case of the positive polarity. ${ }^{13}$ Nevertheless, in both cases, the wave activity develops always in the frequency domain $\omega<\nu_{d n}$ (here $\nu_{d n}$ being the dust-neutral collision frequency) with maximal values $(\omega / 2 \pi)_{\max } \sim 10 \mathrm{~Hz}$. This means that the camera frame rate of the recording at $70 \mathrm{fps}$ (the Nyquist frequency is $35 \mathrm{~Hz}$ ) allows us to avoid any aliasing of wave signals.

One of the fundamental modes in dusty plasmas is the dust acoustic wave which obeys the dispersion law $\omega \approx k u_{d a}$ in the long wavelength range $\left(k \lambda_{D i} \gg 1\right)$. Here, the standard notations of the dust acoustic velocity $u_{d a}=\omega_{p d} \lambda_{D i}$ and plasma frequency $\omega_{p d}$ have been used. One may wonder whether this mode can be invoked for the explanation of the observed dust density perturbations. The wave experiment, however, has been performed in the collisional plasmas, so the standard DAW solution could appear only at wave numbers $k$ $\gg \nu_{d n} / 2 u_{d a}$. The dust acoustic velocity estimations given in Ref. 13 lead to $u_{d a} \sim 1.7 \mathrm{~cm} / \mathrm{s}$. Such a number provides the lower cutoff of the DAW existence at $k>30 \mathrm{~cm}^{-1}$. Therefore, it becomes clear that we cannot attribute the observed mode dispersion behavior to the ordinary DAW, and the wave properties demand a physical explanation. Below we show that the positive and negative discharge electric field

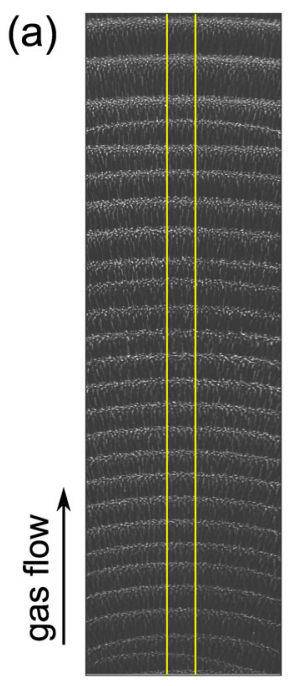

(b)

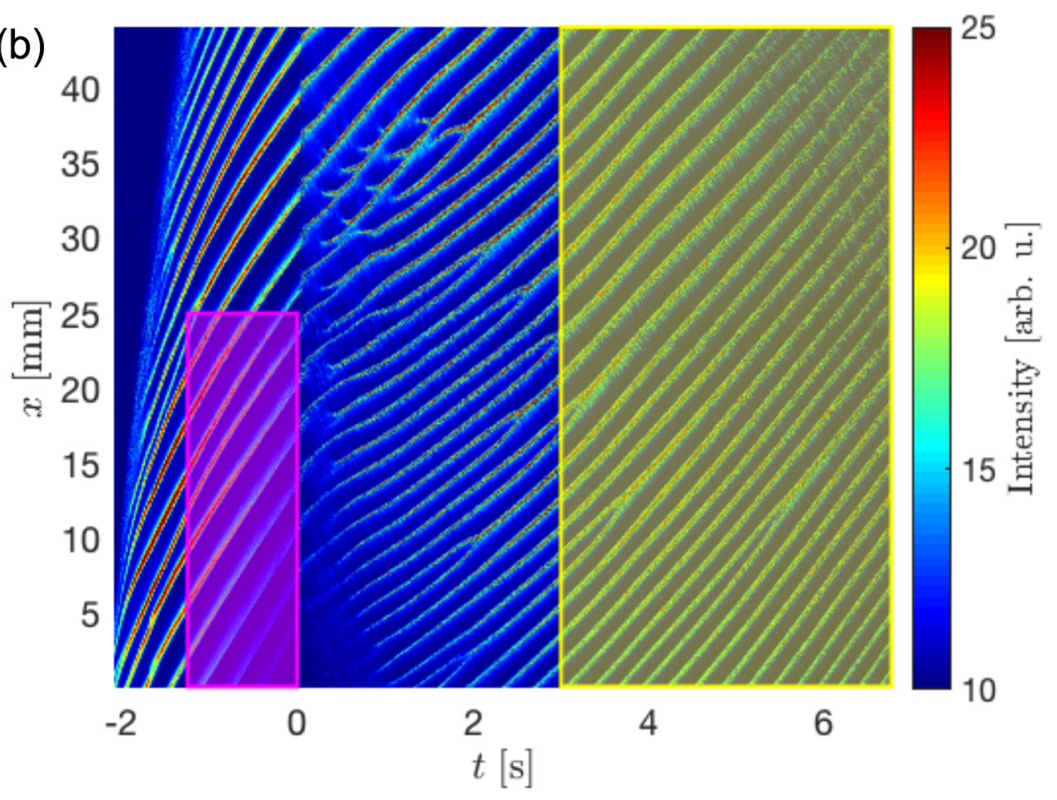

FIG. 1. (a) Image of dust density waves obtained by combining images from two video cameras in the polarity reversal experiment.. ${ }^{13}$ The region between the yellow lines has been used to produce a spatiotemporal pattern of the scattered light intensity $J(x, t)(b)$. Point $t=0$ indicates the moment of polarity reversal. Space-time domains, where Fourier transforms have been performed, are marked by magenta and yellow rectangles for negative and positive polarity, respectively. 

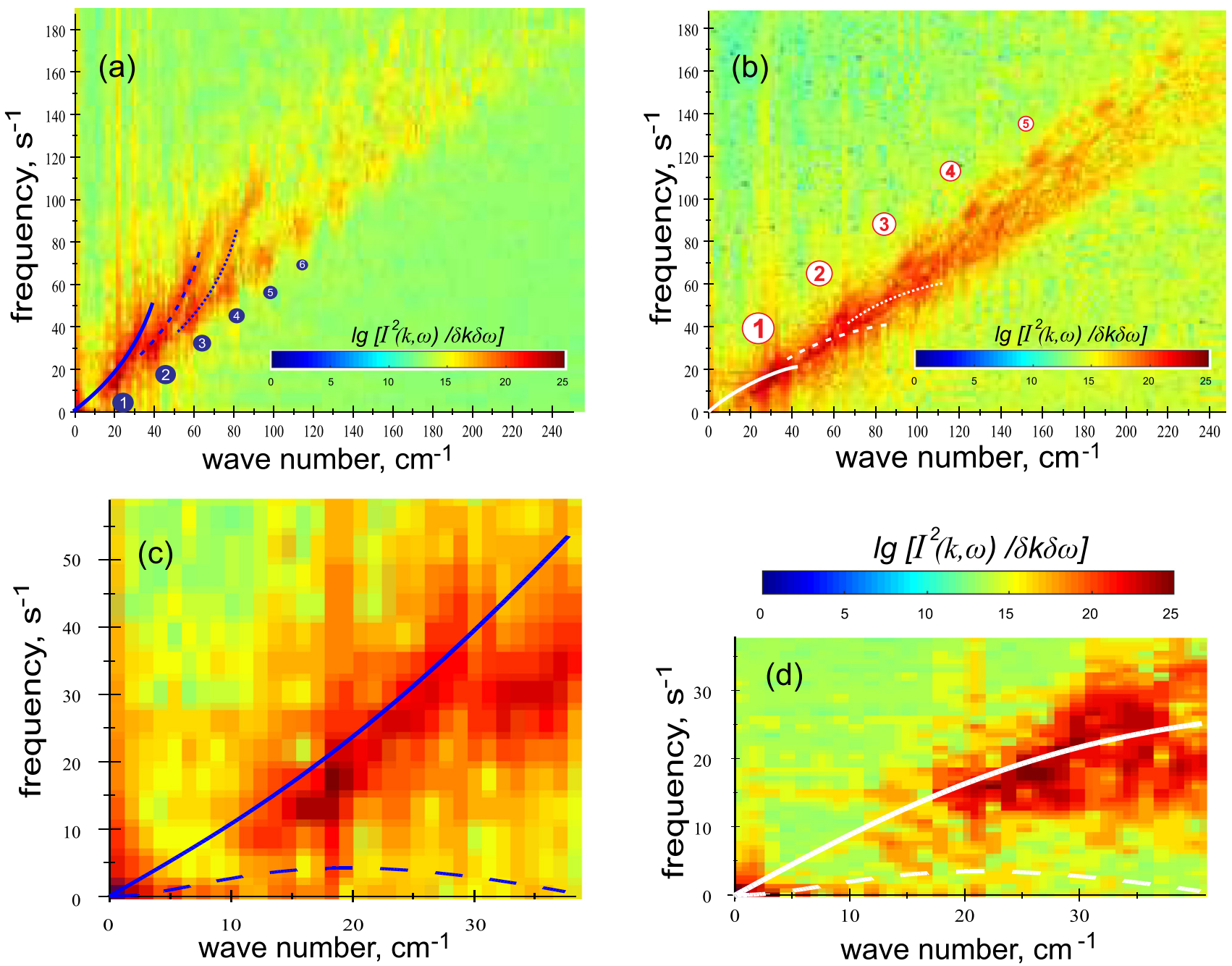

FIG. 2. Fluctuation spectra $R^{2}(k, \omega)$ of dust density waves image intensity for the polarity reversal experiment,,$^{13}$ calculated from the respective spatiotemporal spectrogram regions indicated in Fig. 1: (a) at negative discharge polarity; (b) at positive discharge polarity. The periodic weak features in both spectra indicated by numbers are attributed to higher harmonics generated by nonlinear effects. The theoretically found fundamental mode (9) and its first harmonics are plotted by solid, dashed, and dotted lines, respectively for negative (a) and positive (b) polarities. Neighborhood of the maxima of the $I^{2}(k, \omega)$ spectra for negative (c) and positive polarity (d). The blue/white solid curves illustrate the respective theoretical dispersion laws (9), while the dashed curve describes the instability growth rate (10) in the parameter space of the dust mode experiment as explained in the text.

on the order of a few $\mathrm{V} / \mathrm{cm}$, and associated microparticle drifts in combination with the gas flow can provide two unstable dust density modes which develop at the small $k$-low frequency $\omega$ domain.

\section{PLASMA AND DUST PARAMETERS}

In Table I, we have summarized the plasma and dust parameters from the experiment. ${ }^{13}$ It is important to notice that the plasma parameter estimations have been made in the absence of dust grains. It can be easily shown from the appropriate momentum equations that in stationary limit a zero-order electric field $E_{0}$ imparts the equilibrium drifts to the electrons $(\alpha=e)$, ions $(\alpha=i)$ and dust particles $(\alpha=d)$

$$
u_{0 \alpha}=-\frac{q_{\alpha}}{\nu_{\alpha, n} m_{\alpha}} E_{0},
$$

where $m_{\alpha}$ and $q_{\alpha}$ refer to the plasma particle masses and charges, and $q_{e}$ $=-q_{i}=e$ for the plasma electrons and ions, while for the dust grains $q_{d}$ $=e Z_{d}$. The quantities $\nu_{\alpha n}$ describe collisions with the neutral gas. For plasma electrons and ions, these quantities are usually defined by $\nu_{\alpha n} \simeq n_{n} \sigma_{\alpha n} \nu_{T \alpha}$, where $n_{n}$ is the neutral number density, $\sigma_{\alpha n}$ denotes the collisional cross section for a neutral gas, and $v_{T \alpha}=\sqrt{T_{\alpha} / m_{\alpha}}$ is the thermal velocity with $T_{\alpha}$ being the kinetic temperature of plasma species $\alpha$. The momentum transfer frequency in dust-neutral collisions can be described by the standard Epstein theory ${ }^{15}$ leading to $\nu_{d n}=(8 \sqrt{2 \pi})$ 3) $a^{2} n_{n} m_{n} v_{T n} / m_{d}$, where $m_{n}, T_{n}$, and $v_{T n}=\sqrt{T_{n} / m_{n}}$ are the mass, temperature, and thermal velocity of neutrals, respectively. Note that the expression for the drift velocities (1) does not include the drag due to the ion-dust interaction. Below, we discuss this issue in more detail. 
TABLE I. Characteristics of the dusty plasmas in the PK-4 setup. ${ }^{\text {a }}$

\begin{tabular}{lc}
\hline \hline DC current, $j_{d c}(\mathrm{~mA})^{* \mathrm{~b}}$ & $-0.5 /+0.5$ \\
Pressure, $P(\mathrm{~Pa})$ & 40 \\
Ion density, $n_{0 i}\left(\mathrm{~cm}^{-3}\right)$ & $1.3 \times 10^{8}$ \\
Electron temperature, $T_{e}(\mathrm{eV})$ & 8.6 \\
Ion temperature, $T_{i}(\mathrm{eV})$ & 0.03 \\
Particle size, $a(\mu \mathrm{m})$ & 1.7 \\
Particle charge number, $Z_{d}$ & $\sim 2 \times 10^{3}$ \\
Dust number density, $n_{0 d}\left(\mathrm{~cm}^{-3}\right)$ & $\sim 10^{5}$ \\
Ion collision frequency $\nu_{i n}\left(\mathrm{~s}^{-1}\right)$ & $4 \times 10^{6}$ \\
Dust collision frequency $\nu_{d n}\left(\mathrm{~s}^{-1}\right)$ & 90 \\
Electric field, $E_{0}(\mathrm{~V} / \mathrm{cm})^{*}$ & $\sim-2.4 /+2.4$ \\
Ion thermal Mach number, $M_{i}^{*}$ & $\sim-0.7 /+0.7$ \\
Average cloud dust velocity, $(\mathrm{cm} / \mathrm{s})^{*}$ & $\sim+3.3 / \gtrsim-1.9$ \\
\hline \hline
\end{tabular}

${ }^{\mathrm{a}}$ Numerical data are adopted from Ref. 13.

${ }^{\mathrm{b}}$ Data marked by ${ }^{*}$ given for negative/positive polarity, respectively.

Normalizing the velocities (1) for electrons and ions to a respective thermal speed, we obtain the ratio of the thermal Mach numbers $M_{e} / M_{i}=\sigma_{i n} /\left(\sigma_{e n} \tau\right)$ where $\tau$ is an electron-to-ion temperature ratio $T_{e} / T_{i}$. Typically, the ratio of the cross sections yields $\sigma_{i n} / \sigma_{e n} \sim 10$, while for our experimental conditions $\tau \sim 300$; hence, the ion drift effect is much stronger than that for the electrons, which are further considered as obeying a Boltzmann type distribution.

Two cases are to be distinguished concerning the direction of the axial electric field $E_{0}$. For negative polarity, the ions have negative drift velocity $u_{0 i}<0\left(M_{i}<0\right)$, while the microparticle drift velocity, $u_{0 d}$, is positive. Moreover, in this case, $u_{0 d}$ and the neutral gas velocity, $V_{g a s}$, are in the same direction, providing the positive zero-order streaming velocity for the dust grains $V_{0 d}^{(-)}$. In contrast, at the positive polarity, the ion drift velocity is positive $u_{0 i}>0\left(M_{i}>0\right)$, the negative drift velocity $u_{0 d}$ and neutral gas velocity, $V_{\text {gas }}$, are reversed, and the net zero-order streaming velocity $V_{0 d}^{(+)}$is negative. The microparticle drift velocity can be written as

$$
V_{0 d}^{(\mp)}= \pm \frac{e Z_{d}}{\nu_{d n} m_{d}}\left|E_{0}\right|+V_{g a s},
$$

where the indices $\mp$ refer to the negative or positive polarity, respectively.

Addressing the problem of the equilibrium drift velocities, we have neglected the force related to the momentum transfer from a flowing plasma to the charged grains and vice versa. To justify this assumption, we consider a ratio of the ion-neutral to ion-dust collisional frequency in the parameter space relevant for the wave experiment. Here, we introduce the ion-dust momentum-transfer frequency as $\nu_{i d} \simeq n_{0 d}\left(\pi \Delta^{2} / 4\right) v_{T i}$, where $n_{0 d}$ and $\Delta \simeq\left(n_{0 d}\right)^{-1 / 3}$ are the equilibrium dust number density and mean interparticle distance. Such an expression for $\nu_{i d}$ is a reasonable approximation in dense dust clouds, when the characteristic length of ion dust interactions $r_{C}$ $=Z_{d} e^{2} / T_{i}$ is larger or on the order of the interparticle distance $\Delta .^{16}$ Calculating the ratio of two collisional frequencies gives $\nu_{\text {in }} / \nu_{i d}=\left(n_{n} / n_{d 0}\right)\left(4 \sigma_{\text {in }} / \pi \Delta^{2}\right) \sim 10$, thus showing that the ion drag force is one order of magnitude smaller than the neural drag and for simplicity can be omitted.
Finally, the ion mean free path $l_{\text {in }}=1 /\left(n_{n} \sigma_{\text {in }}\right) \sim 10^{-2} \mathrm{~cm}$ is on the order of the plasma (ion) Debye length $\lambda_{D i} \simeq 1.3 \times 10^{-2} \mathrm{~cm}$ being significantly smaller than the wavelength of the excited dust density perturbations $\lambda \gtrsim 0.1 \mathrm{~cm}$. This implies that the ions are in the highly collisional regime, and the ion charge exchange collisions can result in significant reduction of the equilibrium particle charge in comparison to that given by the standard OML (Orbit Motion Limited) theory. ${ }^{17-21}$ Using the calculations of the equilibrium grain charge accounting for the collisional effect in the parameter space of the PK-4 device, one can expect the charge number of the isolated particle, $Z_{d}$ lying in the range between $2 \times 10^{3}$ and $2.8 \times 10^{3}$ at the pressure $p=40 \mathrm{~Pa}$ in neon plasma. ${ }^{22}$ The real dust charge can, however, be even lower due to the large dust number density.

\section{LINEAR MODE FORMALISM}

Within the electrostatic formalism, the low-frequency dust-density mode is governed by the dispersion relation that in terms of the plasma susceptibilities reads as

$$
1+\chi_{e}+\chi_{i}+\chi_{d}=0 .
$$

The susceptibility of the Boltzmann distributed electrons is standard $\chi_{e}=1 / k^{2} \lambda_{D e}^{2}$, where $k$ is the wave number and $\lambda_{D e}$ stands for the electron Debye length.

The dust susceptibility derived from a fluid model accounts for a dust drift velocity $V_{0 d}=V_{0 d}^{( \pm)}$in the external electric fields, and is given by

$$
\chi_{d}=-\frac{\omega_{p d}^{2}}{\left(\omega-k V_{0 d}\right)\left(\omega-k V_{0 d}+i \nu_{d n}\right)} .
$$

The latter expression does not include the effect of the dust component pressure. ${ }^{23,24}$ We have verified that it does not play a major role under the conditions investigated.

Finally, for the ion susceptibility, we adopt the expression following from the kinetic theory accounting for the strongly collisional case. Dealing with the subthermal ion drift, $\left|M_{i}\right| \leqq 1$, and small wave number limit $k \leqq\left|M_{i}\right| \nu_{\text {in }} / V_{T i}$ yields

$$
\chi_{i} \simeq i \frac{\omega_{p i}^{2}}{\nu_{i n}\left(\omega-k u_{0 i}\right)}\left(1-i \frac{k^{2} V_{T i}^{2}}{\nu_{i n}\left(\omega-k u_{0 i}\right)}\right) .
$$

Here the plasma frequency of various species is defined through $\omega_{p \alpha}=\sqrt{4 \pi q_{\alpha}^{2} n_{0 \alpha} / m_{\alpha}}$ with $n_{0 \alpha}$ being the zero-order plasma/dust density. Neglecting small $\omega$ (compared to the large Doppler shifted term $\left(k u_{0 i}\right)$, ) the expression for $\chi_{i}$ can be further simplified to

$$
\chi_{i} \simeq \frac{\omega_{p i}^{2}}{\nu_{i n}^{2} M_{i}^{2}}-i \frac{\omega_{p i}^{2}}{\nu_{i n} k u_{0 i}} .
$$

Inserting $\chi_{\alpha}$ in (3) yields

$$
1+\frac{1}{k^{2} \lambda_{D e}^{2}}+\frac{\omega_{p i}^{2}}{\nu_{i n}^{2} M_{i}^{2}}-i \frac{\omega_{p i}^{2}}{M_{i} k v_{T i} \nu_{i n}}-\frac{\omega_{p d}^{2}}{\Omega\left(\Omega+i \nu_{d n}\right)}=0
$$

with the Doppler shifted frequency $\Omega=\omega-k V_{0 d}$.

The dispersion equation (7) has two solutions for the real part of the frequency 
$\Omega= \pm \frac{1}{\sqrt{2}}\left[\frac{\omega_{p d}^{2} A}{A^{2}+B^{2} / k^{2}}-\frac{\nu_{d n}^{2}}{4}+\left(\frac{\nu_{d n}^{4}}{16}+\frac{\left(\omega_{p d}^{2}-0.5 A \nu_{d n}^{2}\right) \omega_{p d}^{2}}{A^{2}+B^{2} / k^{2}}\right)^{1 / 2}\right]^{1 / 2}$,

where $A=1+1 / k^{2} \lambda_{D e}^{2}+\omega_{p i}^{2} / \nu_{i}^{2} M_{i}^{2}$ and $B=\omega_{p i}^{2} / M_{i} v_{T i} \nu_{i}$. Two signs in Eq. (8) are unambiguously determined by the direction of the ion flow: "plus" corresponds to the case $M_{i}>0$ (positive polarity), while $M_{i}<0$ (negative polarity) requires "minus." Note that for nondrifting microparticles only the positive value of $\Omega$ makes sense. A similar low-frequency solution has been considered earlier using kinetic description ${ }^{6}$ and fluid approach, accounting for the dust charge fluctuations $^{25}$ (which are neglected in the present consideration).

Taking into account the microparticle flows (2), one finds two modes (valid as long as $k \ll\left|M_{i}\right| \nu_{\text {in }} / V_{T i} \sim 70 \mathrm{~cm}^{-1}$ ) in the laboratory reference frame

$$
\omega^{(\mp)}=k V_{0 d}^{(\mp)}+\Omega .
$$

Here $V_{0 d}^{(\mp)}$ and $\Omega$ correspond to respective values for negative/positive discharge polarity.

Clearly, the wave solutions (9) are related to microparticle streaming effects at different polarities in the presence of gas flow, which establishes a certain asymmetry in the dust drift velocities [see Eq. (2)]. In the absence of the gas flow, the dust modes would be described by the same dispersion relation (9) with $V_{0 d}^{(\mp)} \rightarrow \pm V_{0}$, where the different signs relate to perturbations propagating along (-) or against $(+)$ the discharge electric field.

Keeping in mind the application of the found solutions (9) to the wave excitation in the polarity reversal experiment, the most interesting case is when two different modes exist in the same discharge plasma. From the measured data, we can make several conclusions about the parameters of dust particles. Examples will be illustrated in Sec. V, but these can only be relevant once we have determined for which conditions the perturbations (9) can be excited.

Both solutions become unstable due to the ion-drift-driven instability, which is analyzed using the dispersion relation (7). The standard stability analysis leads to the following expression for the imaginary part of the frequency:

$$
\begin{aligned}
\omega_{i} \simeq & -\frac{\nu_{d n}}{2}+\frac{1}{\sqrt{2}}\left[\frac{\nu_{d n}^{2}}{4}-\frac{\omega_{p d}^{2} A}{A^{2}+B^{2} / k^{2}}\right. \\
& \left.+\left(\frac{\nu_{d n}^{4}}{16}+\frac{\left(\omega_{p d}^{2}-0.5 A \nu_{d n}^{2}\right) \omega_{p d}^{2}}{A^{2}+B^{2} / k^{2}}\right)^{1 / 2}\right]^{1 / 2} .
\end{aligned}
$$

The waves propagating opposite/along the discharge electric field are unstable, provided that $\omega_{i}$ is positive, and this defines the instability condition as

$$
\frac{\omega_{p d}^{2}}{\nu_{d n}^{2}}>\frac{A\left(A^{2} k^{2}+B^{2}\right)}{B^{2}} .
$$

The terms on the right hand side of (11) satisfy $A^{2} k_{\max } \ll B^{2}$; hence, they lead to the lower limit of the dust plasma frequency $\left(\omega_{p d}\right)_{\min } \simeq \sqrt{A} \nu_{d n} \sim 135 \mathrm{~s}^{-1}$ in the parameter set of Table I. The unstable dust density perturbations can only occur when the dust plasma frequency is above $\left(\omega_{p d}\right)_{\min }$. On the other hand, (11) ensures a restriction on the wave numbers of the unstable perturbations. For any admissible $\omega_{p d}>\left(\omega_{p d}\right)_{\min }$, there will be a window in the wave number domain corresponding to the effectively excited perturbations

$$
\begin{gathered}
\frac{\left(\frac{\omega_{p i}^{2}}{M_{i} \nu_{T i} \nu_{i}}\right)}{\left(1+\frac{\omega_{p i}^{2}}{\nu_{i n}^{2} M_{i}^{2}}\right)^{3 / 2}}\left(\frac{\omega_{p d}^{2}}{\nu_{d n}^{2}}-1-\frac{\omega_{p i}^{2}}{\nu_{i n}^{2} M_{i}^{2}}\right)^{1 / 2} \\
\gtrsim k \gtrsim\left(\frac{\omega_{p d}^{2}}{\nu_{d n}^{2}}-1-\frac{\omega_{p i}^{2}}{\nu_{i n}^{2} M_{i}^{2}}\right)^{-1 / 2} \lambda_{D e}^{-1} .
\end{gathered}
$$

The electron screening length $\lambda_{D e}$ in the right-hand side of (12) must take into account the electron depletion due the presence of the dust particles through the plasma quasineutrality condition. Introducing the Havnes parameter $p=Z_{d} n_{0 d} / n_{0 i}$ yields $n_{0 e}=n_{0 i}(1-p)$ and the definition $\lambda_{D e}=\sqrt{T_{e} /\left(4 \pi e^{2} n_{0 i}(1-p)\right)}$ contains the dust characteristics. Expressing the dust plasma frequency in terms of the Havnes parameter $\omega_{p d}=\sqrt{4 \pi Z_{d} n_{0 i} p / m_{d}} \propto p^{1 / 2}$, we plot in Fig. 3 the instability range (12) in the parameter space $\{k, p\}$. The example has been calculated for the set of parameters presented in Table I and the assumption that the particles have an average equilibrium charge $Z_{d 0} \simeq 2.4 \times 10^{3}$. Figure 3 points out to a considerably high instability threshold in terms of the dust charge density $p \sim 0.6$. For any admissible $p>0.6$, there will be two cutoff values for $k$ according to (12). It is seen that as $p$ approaches unity, the cutoff at small wave numbers gives $k_{1} \sim 1 \mathrm{~cm}^{-1}$, while the upper cutoff increases, yielding a wider range of effectively excited wave numbers. The wave number $k_{2} \gtrsim 40 \mathrm{~cm}^{-1}$, close to the upper limit of the observed $k$-vales in the experiment, requires a Havnes parameter $p \rightarrow 1$. In Fig. 3, we also show the preferentially excited wave number $k_{\max }$

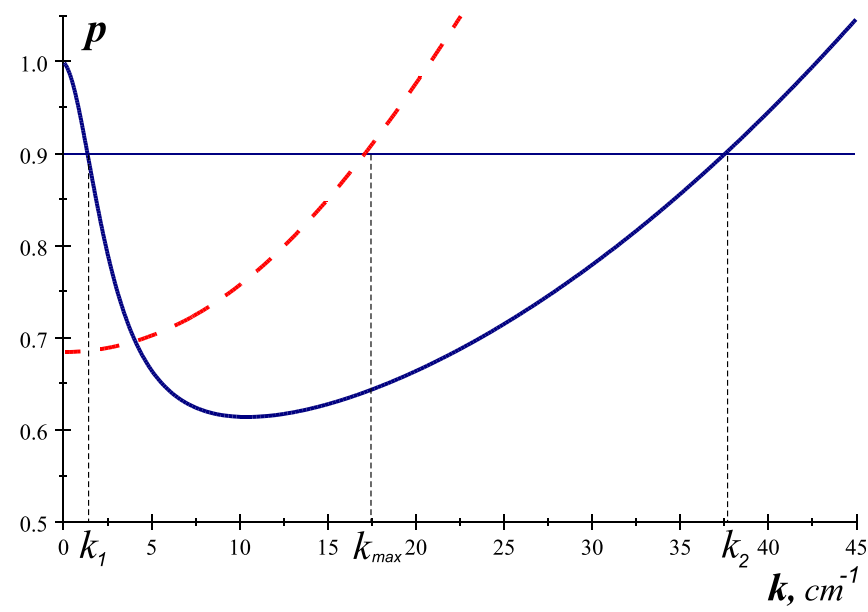

FIG. 3. The instability parameter space in terms of $\{k, p\}$. For any given $p$, the wave numbers $k_{1}$ and $k_{2}$ restrict the interval where the dust density waves can be effectively excited. The dashed curve expresses the preferentially excited wave number $k_{\max }$ as a function of Havnes parameter $p=Z_{d} n_{0 d} d n_{0 i}$. Both calculations have been done for the dust plasma frequency $\omega_{p d}=\sqrt{4 \pi Z_{d} n_{0 i} p / m_{d}}$ with $Z_{d}$ $\simeq 2.4 \times 10^{3}$. Other plasma parameters are as in Table $\mid$. 
defined by the condition $\left(\partial \omega_{i} / \partial k\right)_{k=k_{\max }}=0$ and plotted by the dashed curve. As seen, increasing $p$ gives larger $k_{\max }$ and yields excitation of the dust density waves at smaller wavelengths.

\section{COMPARISON WITH EXPERIMENTS AND IMPLICATIONS FOR PLASMA/DUST DIAGNOSTICS}

We consider the relevance of the found solutions $\omega^{(-)}$and $\omega^{(+)}$ for the experimental dispersion dependencies in Figs. 2(c) and 2(d) from a standpoint of the plasma parameters given in Table I. Taking the average value of the most unstable wave numbers in Figs. 2(c) and 2(d) as $k_{\max } \simeq 22 \mathrm{~cm}^{-1}$, we measure a corresponding local phase speed of the waves. This gives $V_{p h}^{(-)}=\omega^{(-)} / k_{\max } \simeq 1.07 \mathrm{~cm} / \mathrm{s}$ (negative discharge polarity) and $V_{p h}^{(+)}=\omega^{(+)} / k_{\max } \simeq 0.83 \mathrm{~cm} / \mathrm{s}$ (positive polarity). On the other hand, calculating $V_{p h}^{(-)}$and $V_{p h}^{(+)}$from Eq. (9) and eliminating $\Omega$ and $u_{0 d}$ yields for the gas flow velocity $V_{\text {gas }}=\left(V_{p h}^{(+)}+V_{p h}^{(-)}\right) / 2 \simeq 0.95 \mathrm{~cm} / \mathrm{s}$. Furthermore, taking the average cloud velocity observed at the negative polarity $V_{0 d}^{(-)} \simeq 3.3 \mathrm{~cm} / \mathrm{s}$, one immediately finds $u_{0 d} \simeq 2.38 \mathrm{~cm} / \mathrm{s}$ which yields the dust charge $Z_{d} \simeq 1.73 \times 10^{3}$ in the electric field $E_{0} \simeq-2.4 \mathrm{~V} / \mathrm{cm}$. The estimate $Z_{d} \simeq 1.73 \times 10^{3}$ is lower than a predicted value for an isolated grain ${ }^{22}$ but seems to be a reasonable first approximation for the discharge with suspended microparticles. The respective average drift velocity under the positive polarity then becomes $V_{0 d}^{(+)} \simeq-1.4 \mathrm{~cm} / \mathrm{s}$, i.e., the particles move slightly slower than the cloud head $\left(V_{0 d}^{(+)} \simeq-1.9 \mathrm{~cm} / \mathrm{s}\right)$ according to the data of Ref. 13 .

In a similar way, the difference of two phase velocities $\left(V_{p h}^{(-)}\right.$ $\left.-V_{p h}^{(+)}\right)$at $k=k_{\max }$ leads to

$$
\frac{e Z_{d}}{\nu_{d n} m_{d}} E_{0}-\frac{\Omega\left(k_{\max }\right)}{k_{\max }} \simeq 0.12 .
$$

For $k \gg 1$, the quantity $A$ is simplified to $A \simeq 1+\omega_{p i}^{2} / \nu_{i}^{2} M_{i}^{2}$ and expressions (8)-(10) become a function of only $\omega_{p d}^{2}$ since other parameters are given. Hence, Eq. (13) enacts a certain relation between $Z_{d}$ and $\omega_{p d}$. Later, we use (13) for independent verification of the obtained dust characteristics.

To plot the dispersion curves described by Eq. (9) requires the value of the dust plasma frequency, in which the particle charge $Z_{d}$ and dust number density $n_{0 d}$ are coupled together. On the other hand, $n_{0 d}$ remains often one of the most difficulty measured quantities in dense dust clouds. We can determine the microparticle density $n_{0 d}$ independently. Solving $\partial \omega_{i} / \partial k=0$ gives the preferentially excited wave number $k=k_{\max }$. In Fig. 4, the value $k_{\max }$ is shown as a function of $\omega_{p d}$. For any chosen $k_{\max }$, there will be one crossover value for the dust plasma frequency. Taking $k_{\max } \simeq 22 \mathrm{~cm}^{-1}$ gives $\omega_{p d} \simeq 166 \mathrm{~s}^{-1}$. This value exceeds $\left(\omega_{p d}\right)_{\min } \sim 135 \mathrm{~s}^{-1}$ and, on the other hand, provides the lower and upper limits of the wave numbers $k_{1} \gtrsim 1 \mathrm{~cm}^{-1}$ and $k_{2} \sim 40 \mathrm{~cm}^{-1}$ well corresponding to the observed range of the excited wave numbers in Figs. 2(d) and 2(c). The estimates $Z_{d}$ $\simeq 1.73 \times 10^{3}$ and $\omega_{p d} \simeq 166 \mathrm{~s}^{-1}$ yield the dust number density $n_{0 d}$ $\simeq 9.7 \times 10^{4} \mathrm{~cm}^{-3}$, close to the average value in Ref. 13 . Note that the obtained dust parameters lead to the Havnes parameter slightly above unity at the ion density calculated in the absence of microparticles (Table I). It is reasonable to assume that in reality, a discharge plasma modified by charged microparticles would have higher values of $n_{0 i}{ }^{26}$

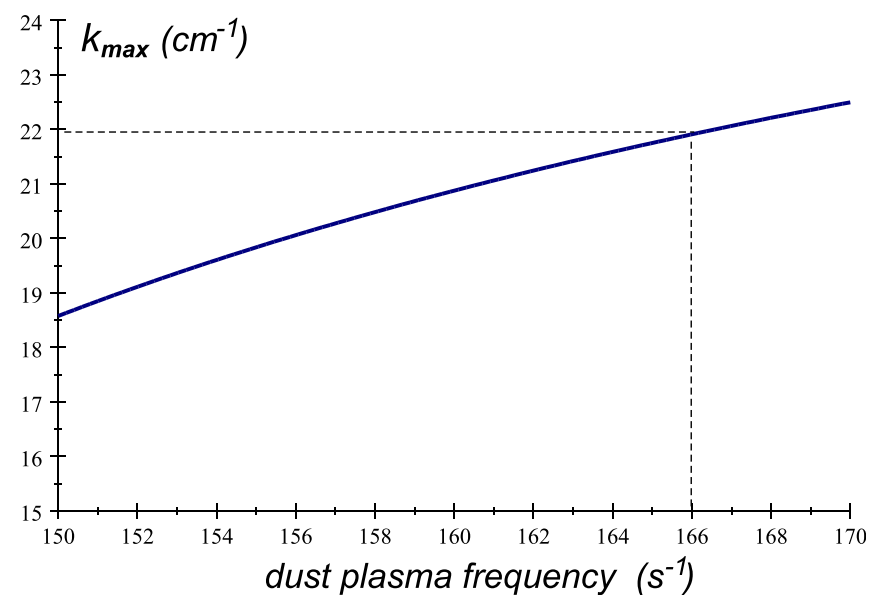

FIG. 4. The wave number $k_{\max }$ corresponding to the maximal growth rate of the drift-driven instability vs the dust plasma frequency for the parameter set of Table I.

hence the Havnes parameter would obey $p \lesssim 1$. Now, the obtained numbers for $Z_{d}$ and $\omega_{p d}$ have to be checked using the relation (13). Inserting $\omega_{p d} \simeq 166 \mathrm{~s}^{-1}$ into $\Omega\left(k_{\max }\right)$ and solving Eq. (13) numerically leads to $Z_{d} \simeq 1.72 \times 10^{3}$ perfectly confirming the dust charge value employed above.

In Figs. 2(d) and 2(c), we show two dispersion curves resulting from the relations (9) (solid lines) and (10) (dashed lines) which are calculated for the obtained values of $\omega_{p d} \simeq 166 \mathrm{~s}^{-1}, V_{0 d}^{(-)} \simeq 3.3 \mathrm{~cm} / \mathrm{s}$, and $V_{0 d}^{(+)} \simeq-1.4 \mathrm{~cm} / \mathrm{s}$. Other parameters correspond to the data set of Table I. As seen, both plots $\omega^{(-)}(k)$ and $\omega^{(+)}(k)$ quite reasonably match with the respective parts of the space-time Fourier spectra measured at different discharge polarities in the long wavelength range. Therefore, the considered perturbations are relevant candidates for the explanation of the observed self-excited waves in the microparticle flows. Some discrepancies can be attributed to the fact that our modeling deals with the homogeneous complex plasma while the real case includes variations of the background plasma/dust parameters ${ }^{13}$ as dust drift velocity, dust charge density, gradients of the discharge electric field, etc. These factors definitively cause the broadening of the measured fluctuation spectra shown in Fig. 2.

\section{CONCLUSIONS}

We have studied the dust density waves propagating in the flowing complex plasma under the conditions close to those observed in the polarity reversal experiment. ${ }^{13}$ We consider a related plasma model, suggesting that a primary process responsible for the wave excitation is the ion-streaming instability. Such an instability can provide energy for excitation of dust density perturbations in the lowfrequency-small wave number domain in collisional complex plasmas. Two wave modes excited at negative/positive polarity propagate in the same direction-along the gas flow. The real and imaginary parts of the linear dispersion relation reasonably agree with the respective parts of the measured Fourier spectra as shown in Figs. 2(c) and 2(d). Therefore, theoretically found compressional dust modes can qualitatively explain the wave properties observed at both discharge polarities in the PK-4 setup: The range of excited wave numbers $15 \mathrm{~cm}^{-1} \lesssim k \lesssim 35 \mathrm{~cm}^{-1}$, wave number cutoffs $k_{1} \sim 1-5 \mathrm{~cm}^{-1}$, the 
most unstable wave numbers $k_{\max } \sim 20 \mathrm{~cm}^{-1}$; and different phase velocities of the two modes. The dispersions of both dust density waves are close to acoustic type and, thus, may provide the resonant interactions between the fundamental mode and its harmonics generated by nonlinear effects, thus explaining the extension of the enhanced wave energy fluctuations up to $k \sim 100 \mathrm{~cm}^{-1}$ [Figs. 2(a) and 2(b)].

Comparison of the theoretical results and experimental spectra allows us to quantify some important parameters like the microparticle charge, dust number density, and gas flow velocity. Our studies indicate that the value of the dust charge density (Havnes parameter) introduces the instability threshold and defines a range of self-excited wave numbers, and a considerably wide range of excitation requires $p \rightarrow 1$ (Fig. 3). One can conclude, therefore, that the Havnes parameter may be a key factor allowing for the excitation of the dust density modes. The high threshold on the dust charge density can introduce a main limitation in the experimental observations of the dust density modes under the PK-4 discharge conditions. Future wave experiments in the PK-4 setup can elucidate the role of the dust charge density in the development of collective modes under various conditions.

\section{ACKNOWLEDGMENTS}

All authors greatly acknowledge the joint ESA/Roscosmos "Experiment Plasmakristall-4" on board the International Space Station. The authors also thank V. Nosenko for fruitful discussions of the obtained results.

\section{REFERENCES}

${ }^{\mathbf{T}}$ R. L. Merlino, “25 years of dust acoustic waves," J. Plasma Phys. 80, 773-786 (2014).

${ }^{2}$ V. V. Yaroshenko, F. Verheest, and G. Morfill, Astron. Astrophys. 461, 385-391 (2007)

${ }^{3}$ F. Verheest and V. Yaroshenko, Astron. Astrophys. 503, 683-690 (2009).

${ }^{4}$ N. N. Rao, P. K. Shukla, and M. Y. Yu, Planet Space Sci. 38, 543 (1990).

${ }^{5}$ M. Rosenberg, Planets Space Sci. 41, 229 (1993).

${ }^{6}$ M. Rosenberg, J. Vac. Sci. Technol., A 14, 631 (1996).

${ }^{7}$ V. E. Fortov, A. G. Khrapak, S. A. Khrapak, V. I. Molotkov, A. P. Nefedov, O. F. Petrov, and V. M. Torchinsky, Phys. Plasmas 5, 1374 (2000).

${ }^{8}$ A. Piel, O. Arp, M. Klindworth, and A. Melzer, Phys. Rev. E 77, 026407 (2008).
${ }^{9}$ S. A. Khrapak, D. Samsonov, G. Morfill, H. Thomas, V. Yaroshenko, H. Rothermel, T. Hagl, V. Fortov, A. Nefedov, V. Molotkov, O. Petrov, A. Lipaev, A. Ivanov, and Y. Baturin, Phys. Plasmas 10, 1 (2003).

${ }^{10}$ M. Rosenberg, E. Thomas, Jr., and R. L. Merlino, Phys. Plasmas 15, 073701 (2008).

${ }^{11}$ V. V. Yaroshenko, B. M. Annaratone, S. A. Khrapak, H. M. Thomas, G. E. Morfill, V. E. Fortov, A. M. Lipaev, V. I. Molotkov, O. F. Petrov, A. I. Ivanov, and M. V. Turin, Phys. Rev. E 69, 066401 (2004).

${ }^{12}$ V. Nosenko, S. K. Zhdanov, S. H. Kim, J. R. Heinrich, R. L. Merlino, and G. E. Morfill, Europhys. Lett. 88, 65001 (2009).

${ }^{13}$ S. Jaiswal, M. Y. Pustylnik, S. Zhdanov, H. M. Thomas, A. M. Lipaev, A. D. Usachev, V. I. Molotkov, V. E. Fortov, M. H. Thoma, and O. V. Novitskii, Phys. Plasmas 25, 083705 (2018).

${ }^{14}$ M. Y. Pustylnik, M. A. Fink, V. Nosenko, T. Antonova, T. Hagl, H. M. Thomas, A. V. Zobnin, A. M. Lipaev, A. D. Usachev, V. I. Molotkov, O F. Petrov, V. E. Fortov, C. Rau, C. Deysenroth, S. Albrecht, M. Kretschmer, M. H. Thoma, G. E. Morfill, R. Seurig, A. Stettner, V. A. Alyamovskaya, A. Orr, E. Kufner, E. G. Lavrenko, G. I. Padalka, E. O. Serova, A. M. Samokutyayev, and S. Christoforetti, Rev. Sci. Instrum. 87, 093505 (2016).

${ }^{15}$ P. S. Epstein, Phys. Rev. 23, 710-733 (1924).

${ }^{16}$ V. V. Yaroshenko, S. A. Khrapak, and G. E. Morfill, Phys. Plasmas 20, 043703 (2013).

${ }^{17}$ A. V. Zobnin, A. P. Nefedov, V. A. Sinel'shchikov, and V. E. Fortov, JETP 91, $483(2000)$

${ }^{18}$ M. Lampe, V. Gavrishchaka, G. Ganguli, and G. Joyce, Phys. Rev. Lett. 86, 5278 (2001).

${ }^{19}$ S. Ratynskaia, S. Khrapak, A. Zobnin, M. H. Thoma, M. Kretschmer, A. Usachev, V. Yaroshenko, R. A. Quinn, G. E. Morfill, O. Petrov, and V. Fortov, Phys. Rev. Lett. 93, 085001 (2004).

${ }^{20}$ S. A. Khrapak, S. V. Ratynskaia, A. V. Zobnin, A. D. Usachev, V. V. Yaroshenko, M. H. Thoma, M. Kretschmer, H. Höfner, G. E. Morfill, O. F. Petrov, and V. E. Fortov, Phys. Rev. E 72, 016406 (2005).

${ }^{21}$ S. A. Khrapak, G. E. Morfill, A. G. Khrapak, and L. G. D'yachkov, Phys. Plasmas 13, 052114 (2006).

${ }^{22}$ T. Antonova, S. A. Khrapak, M. Y. Pustylnik, M. Rubin-Zuzic, H. Thomas, A. M. Lipaev, A. D. Usachev, V. I. Molotkov, and M. H. Thoma, "Particle charge in PK-4 dc discharge from ground-based and and microgravity experiments," Phys. Plasmas (unpublished).

${ }^{23}$ S. A. Khrapak, Plasma Phys. Controlled Fusion 58, 014022 (2015).

${ }^{24}$ S. A. Khrapak and H. M. Thomas, Phys. Rev. E 91, 033110 (2015).

${ }^{25}$ S. Khrapak and V. Yaroshenko, Phys. Plasmas 10, 4616-4621 (2003).

${ }^{26}$ A. D. Usachev, A. Zobnin, O. Petrov, V. Fortov, M. Thoma, H. Höfner, M. Fink, A. Ivlev, and G. Morfill, New J. Phys. 16, 053028 (2014). 